\title{
Hamilton-Jacobi Separable, Axisymmetric, Perfect-fluid Solutions of Einstein's Equations
}

\author{
Sotirios Bonanos
}

N.R.C. Democritos, Aghia Paraskevi, Attiki, Greece

\begin{abstract}
In this paper we examine the Einstein equations with a perfect fluid source under the assumptions of (i) axial symmetry and timeindependence, (ii) uniform rotation of the fluid about the symmetry axis, and (iii) separability of the Hamilton-Jacobi equation for the null geodesics of the space. These assumptions are made in an attempt to generalize the results of a similar investigation by Carter for the source-free case.

We first extend Carter's results by showing that his additional assumption of separability of the wave equation is unnecessary, it being a consequence of the field equations.

When the density of the fluid is non-zero, we are led to a particular solution discovered by Wahlquist, or to more symmetrical interior solutions with spherical equipressure surfaces. Except for the case of no rotation, these solutions cannot be matched to asymptotically flat exteriors.
\end{abstract}

\section{Introduction}

In a 1968 paper [1], Carter examined spaces with a two-parameter Abelian isometry group in which the Hamilton-Jacobi equation for the geodesics separates. While the first assumption (two-parameter Abelian isometry group) is readily interpreted physically as restricting the study to stationary and axisymmetric spacetimes, the second assumption $(\mathrm{H}-\mathrm{J}$ separability) has no simple physical justification. It is made, as Carter admits, in order to obtain sufficiently strong restrictions to make a detailed study possible.

In the same spirit, we use, in this paper, a weaker form of Carter's separability ansatz as a simplifying tool in the study of rotating fluid masses. We find that the only solution having an isometry group with fewer than 3 parameters is one discovered by Wahlquist starting from different assumptions [2]. We also obtain other solutions having spherical symmetry. None of these solutions, however, can represent a finite, isolated object in an asymptotically flat space, except in the limit of no rotation. 
Another result of this paper is a demonstration of the fact that the separability of the wave equation, which Carter assumes explicitly, follows from his other assumptions and the field equations.

\section{The Symmetry and Separability Conditions}

In this section we follow closely Carter's paper [1] in deducing the form of the metric from the symmetry and separability assumptions.

Our symmetry assumptions of time independence, axial symmetry and uniform rotation are equivalent to Carter's assumptions I (invariance under a two-parameter Abelian isometry group) and II (invertibility of the symmetry group). In reference [3] it is shown that the constancy of the angular velocity of the fluid guarantees II. These symmetry assumptions, though materially simplifying the problem, do not restrict it to unrealistic situations: the stationary state of a rotating object must be axisymmetric, and equilibrium against transfer of angular momentum requires that the angular velocity of rotation be constant throughout the star [4].

The essential restriction comes from the requirement that the HamiltonJacobi equation for the null geodesics of the space be soluble by separation of variables in such a way as to determine a canonical orthonormal frame ${ }^{1}$. To the extent that we demand separability for the null geodesics only, our separability requirement is weaker than the corresponding one of Carter who demands separability for all geodesics (his condition IV). The effect on the metric is to allow an extra arbitrary function as a conformal factor. We can thus use Carter's form of the metric ([1], Eq. (77)) and write

$$
d s^{2}=\frac{1}{R^{2}}\left\{\frac{\Lambda(Q d t-P d \varphi)^{2}-M(B d t-A d \varphi)^{2}}{W}-W\left(\frac{d \lambda^{2}}{\Lambda}+\frac{d \mu^{2}}{M}\right)\right\},
$$

where $t$ and $\varphi$ are the two ignorable coordinates corresponding to the two symmetries (time translation and rotation about the axis), $\lambda$ and $\mu$ are the remaining two coordinates in terms of which the separation is possible, $A, B, \Lambda$ are functions of $\lambda$ only, $P, Q, M$ are functions of $\mu$ only, $W=A Q-B P$, and $R$ arbitrary. Carter's stronger separability condition is satisfied if the conformal factor $R$ is given by $R^{2}=W / U$, where $U$ is the sum of a function of $\lambda$ and a function of $\mu$.

In writing (1) we have abandoned Carter's condensed notation with indices preferring to use a different letter for each function entering the metric. The following table translates the notation of this paper to that of Carter's paper [1].

\begin{tabular}{|c|c|c|c|c|c|c|c|c|c|c|c|}
\hline \multirow[b]{2}{*}{ This paper } & \multicolumn{4}{|c|}{ Coordinates } & \multicolumn{7}{|c|}{ Metric functions } \\
\hline & $t$ & $\varphi$ & $\lambda$ & $\mu$ & $\Lambda$ & $M$ & $A$ & $B$ & $Q$ & $P$ & $W$ \\
\hline Paper $[1]$ & $\varphi^{-2}$ & $\varphi^{2}$ & $\lambda^{1}$ & $\lambda^{-1}$ & $\Delta_{1}$ & $\Delta_{-1}$ & $Z_{1}^{1}$ & $Z_{1}^{-1}$ & $Z_{-1}^{-1}$ & $Z_{-1}^{1}$ & $Z$ \\
\hline
\end{tabular}

1 The canonical separation frame is determined by the requirement that, in the H-J equation, "the terms containing derivatives with respect to the ignorable coordinates separate as the sum of two squares each depending on only one of the non-ignorable coordinates" ([1], Condition IV). 
The coordinates $t, \varphi, \lambda, \mu$ are determined by the symmetry and separability conditions up to transformations of the form $\varphi=\varphi^{\prime}+\Omega t$ with $\Omega$ constant, $\lambda=f\left(\lambda^{\prime}\right)$, $\mu=g\left(\mu^{\prime}\right)$. These changes leave the form of the metric (1) unaffected. The freedom in the $\varphi$ coordinate can be removed by requiring that it be a comoving coordinate, i.e., that the fluid 4-velocity have components $u^{\varphi}=u^{\lambda}=u^{\mu}=0, u^{t}=\left(g_{00}\right)^{-1 / 2}$; while the freedom in the $\lambda$ and $\mu$ coordinates can be used to make the functions $Q$ and $B$ constants $^{2}$. Thus, with no coordinate freedom left, the metric becomes

$$
d s^{2}=\frac{1}{R^{2}}\left\{\frac{\Lambda(a d t-P d \varphi)^{2}-M(b d t-A d \varphi)^{2}}{a A-b P}-(a A-b P)\left(\frac{d \lambda^{2}}{\Lambda}+\frac{d \mu^{2}}{M}\right)\right\}
$$

where $a, b$ are constants; $A, \Lambda$ functions of $\lambda ; P, M$ functions of $\mu$; and $R$ arbitrary. The constants $a$ and $b$, if different from zero, can be scaled to 1 . In the particular case when $b=0$, the canonical separation frame coincides with the comoving frame. As $B=0$ in this case, we still have the coordinate freedom $\lambda=f\left(\lambda^{\prime}\right) .(a=0$ is equivalent to $b=0$ up to an interchange of $\lambda$ and $\mu$.)

\section{The Schrödinger Separability Condition}

We use the metric of Equation (1) and define 1-forms $\omega^{i}$ by

$$
\begin{aligned}
\omega^{0} & =\frac{1}{R} \sqrt{\frac{\Lambda}{W}}(Q d t-P d \varphi), & \omega^{1} & =\frac{1}{R} \sqrt{\frac{M}{W}}(A d \varphi-B d t), \\
\omega^{2} & =\frac{1}{R} \sqrt{\frac{W}{\Lambda}} d \lambda, & \omega^{3} & =\frac{1}{R} \sqrt{\frac{W}{M}} d \mu,
\end{aligned}
$$

so that the metric becomes

$$
d s^{2}=\left(\omega^{0}\right)^{2}-\left(\omega^{1}\right)^{2}-\left(\omega^{2}\right)^{2}-\left(\omega^{3}\right)^{2} .
$$

We compute the Ricci tensor in this orthonormal frame, and, after some algebraic manipulation, find that the derivatives of $A, B, P, Q$ in the $R_{23}=0$ equation can be combined into derivatives of $W=A Q-B P$. Thus the field equation $R_{23}=0$ reads simply

$$
2 \frac{R_{\lambda \mu}}{R}+\frac{1}{2} \frac{W_{\lambda \mu}}{W}-\frac{R_{\lambda} W_{\mu}+R_{\mu} W_{\lambda}}{R W}=0,
$$

where the subscripts denote partial derivatives with respect to the indicated variable. Now, Carter's stronger separability condition is equivalent to $R^{2}=W / U$ with $U_{\lambda \mu}=0$. Making this substitution into (5), we find that it can be written as

$$
\left(\log \frac{W}{U}\right)_{\lambda \mu}=0 \text {. }
$$

This is precisely the additional condition that must be satisfied to ensure the separability of the wave equation (compare [1], Eq. (69); Carter's $W$ equals

2 Define primed functions by $R^{2}=(Q B / a b) R^{\prime 2}, \Lambda=\left(B^{2} / b^{2}\right) \Lambda^{\prime}, M=\left(Q^{2} / a^{2}\right) M^{\prime}, A=(B / b) A^{\prime}, P=(Q / a) P^{\prime}$ and primed variables by $d \lambda^{\prime}=(b / B) d \lambda, d \mu^{\prime}=(a / Q) d \mu$; upon substitution in (1) the functions $Q, B$ cancel and we obtain (2) with primes. 
our $W^{2}$ ); it is thus not a separate assumption, but a consequence of the field equations. When the coordinate choice $Q=B=1$ is made, the only solution of (5a) is $W=U$.

\section{The Case $Q=B=1$}

We compute the field equations $G_{j}^{i}=8 \pi\left[(\varrho+p) u^{i} u_{j}-p \delta_{j}^{i}\right]$ where $G_{j}^{i}$ is the Einstein tensor, $\varrho$ the density and $p$ the pressure of the fluid, in the orthonormal frame (3) with $Q=B=1$ and $R$ arbitrary (the case $B=0$ is discussed in the next section). We find that the following linear combinations of the field equations lead to the simplest equivalent set of equations:

$$
\begin{aligned}
& \left\{\left(G_{0}^{0}-G_{2}^{2}\right) u^{1} u_{1}+\left(G_{1}^{1}-G_{3}^{3}\right) u^{0} u_{0}-2 G_{1}^{0} u^{1} u_{0}=0\right\} \quad\left(\frac{R_{\lambda}}{W}\right)_{\lambda}-\left(\frac{R_{\mu}}{W}\right)_{\mu}=0, \\
& \left\{G_{3}^{2}=0\right\} \quad\left(\frac{R_{\lambda}}{W}\right)_{\mu}+\left(\frac{R_{\mu}}{W}\right)_{\lambda}=0, \\
& \left\{\left(G_{0}^{0}-G_{2}^{2}\right) u^{1} u_{1}-\left(G_{1}^{1}-G_{3}^{3}\right) u^{0} u_{0}=0\right\} \quad 2 \frac{R_{\lambda \lambda}+R_{\mu \mu}}{R}=\frac{W_{\lambda \lambda}+W_{\mu \mu}}{W}-\frac{W_{\lambda}^{2}+W_{\mu}^{2}}{W^{2}}, \\
& \left\{G_{1}^{0}=8 \pi(\varrho+p) u^{0} u_{1}\right\} \quad-16 \pi(\varrho+p)=\Theta \frac{R^{4}}{W}\left\{\left(\frac{W_{\lambda}}{R^{2}}\right)_{\lambda}-\left(\frac{W_{\mu}}{R^{2}}\right)_{\mu}\right\}, \\
& \left\{G_{2}^{2}-G_{3}^{3}=0\right\} \quad\left(\frac{\Theta_{\lambda}}{R^{2}}\right)_{\lambda}+\left(\frac{\Theta}{R^{2}}\right)_{\mu}=0, \\
& \left\{G_{2}^{2}+G_{3}^{3}=-16 \pi p\right\} \\
& 16 \pi p=\frac{R^{2}}{2 W}\left(\Lambda^{\prime \prime}+M^{\prime \prime}\right)+\frac{R^{4}}{W}\left[\Lambda\left(\frac{1}{R^{2}}\right)_{\lambda \lambda}+M\left(\frac{1}{R^{2}}\right)_{\mu \mu}\right]-\frac{3 R}{W}\left(\Lambda^{\prime} R_{\lambda}+M^{\prime} R_{\mu}\right) .
\end{aligned}
$$

In writing these equations we have introduced a new function, $\Theta$, defined by $\Theta=(\Lambda-M) /(A-P)$. We observe that, except for (VI), the single-variable functions $A, P, A, M$ enter in these equations in the combinations $W$ and $\Theta$ only. In view of their definitions in terms of $A, P, A, M$, the functions $W$ and $\Theta$ satisfy, in addition to Equations (I-V), the equations $W_{\lambda \mu}=0$ and $(\Theta W)_{\lambda \mu}=0$.

In writing (V) we have made use of (I) and (III) to eliminate $R_{\lambda \lambda}$ and $R_{\mu \mu}$ from $G_{2}^{2}-G_{3}^{3}$.

The integrability conditions of these equations are the equations of motion $T_{; b}^{a b}=0$, which are well known to lead, in the case of uniform rotation in comoving coordinates, to $[5,6]$

$$
2 p_{\alpha}=-(\varrho+p)\left[\log \left(g_{00}\right)\right]_{\alpha} \quad \alpha: \lambda \text { or } \mu .
$$

Equation (6), together with an equation of state of the form $\varrho=\varrho(p)$ imply that both $p$ and $\varrho$ are functions of $g_{00}=\Theta / R^{2}$ in our case.

\footnotetext{
3 The condition that the canonical separation frame is also the canonical frame of the Weyl tensor in the Petrov-Pirani classification ([1], Eq. (137)) turns out to be that the right-hand-side of this equation vanish; in that case the Weyl tensor is type $D$. The solutions to be obtained satisfy this additional requirement and are thus type $D$.
} 
In terms of the complex variable $z=\lambda+i \mu, \bar{z}=\lambda-i \mu$ the field Equations (I)-(V) become:

$(\mathrm{I}+\mathrm{i} I \mathrm{I}) \quad\left(\frac{R_{\bar{z}}}{W}\right)_{\bar{z}}=0$,

$$
2 \frac{R_{z \bar{z}}}{R}=(\log W)_{z \bar{z}}
$$

$$
-8 \pi(\varrho+p)=\Theta \frac{R^{4}}{W}\left\{\left(\frac{W_{z}}{R^{2}}\right)_{z}+\left(\frac{W_{\bar{z}}}{R^{2}}\right)_{\bar{z}}\right\},
$$

$$
\left(\frac{\Theta_{z}}{R^{2}}\right)_{\bar{z}}+\left(\frac{\Theta_{\bar{z}}}{R^{2}}\right)_{z}=0
$$

while the equations $W_{\lambda \mu}=0$ and $(\Theta W)_{\lambda \mu}=0$ become

$$
\begin{aligned}
& W_{z z}=W_{\bar{z} \bar{z}}, \\
& \Theta_{z z}+2 \frac{W_{z}}{W} \Theta_{z}=\Theta_{\bar{z} \bar{z}}+2 \frac{W_{\bar{z}}}{W} \Theta_{\bar{z}} .
\end{aligned}
$$

In this form, the integration of the field equations is straightforward. Equation (7) implies that $R_{\bar{z}} / W$ is a function of $z$ only which, for later convenience, we write as

$$
\frac{R_{\bar{z}}}{W}=\frac{1}{F^{\prime}(z)} \quad \text { where } \quad F^{\prime}(z)=\frac{d}{d z} F(z)
$$

and $F$ is a function of $z$ only. This can be written, using the fact that $W$ and $R$ are real, as

$$
\frac{1}{F^{\prime}(z)} \frac{\partial R}{\partial z}=\frac{W}{F^{\prime}(z) \bar{F}^{\prime}(\bar{z})}=\frac{1}{\bar{F}^{\prime}(\bar{z})} \frac{\partial R}{\partial \bar{z}},
$$

which implies that

(i) $R$ is a function of $(F+\bar{F})$ and

(ii) $W=R^{\prime}(F+\bar{F}) F^{\prime}(z) \bar{F}^{\prime}(\bar{z})$,

$R^{\prime}$ denoting the derivative of $R$ with respect to its argument. To determine $R(F+\bar{F})$ we turn to Equation (8) which becomes

$$
2 \frac{R^{\prime \prime}}{R}=\frac{R^{\prime \prime \prime}}{R^{\prime}}-\left(\frac{R^{\prime \prime}}{R^{\prime}}\right)^{2} .
$$

Two integrations of (15) give $R^{\prime}=d-e R^{3}$, where $d$ and $e$ are constants. Finally, Equation (11), using (14) demands that $e=0$ and that $F(z)$ satisfies $F^{\prime \prime \prime}=-g^{2} F^{\prime}$ where $g^{2}$ is a real constant. Absorbing $d$ into $F$, we see that Equations (7), (8), and (11) are satisfied if

$$
R=F+\bar{F}, \quad W=F^{\prime}(z) \bar{F}^{\prime}(\bar{z}), \quad F^{\prime \prime}+g^{2} F=c
$$

where $g^{2}$ is a real and $c$ a complex constant. 
Using these results in Equation (9) we find

$$
8 \pi(\varrho+p)=2 \Theta R(c+\bar{c}) .
$$

Since we know, from the integrability condition, Equation (6), that $\varrho+p$ is a function of $\Theta / R^{2}$, Equation (17) can only hold if one of the following is true:

(a) $\Theta=$ constant;

(b) $R=$ constant;

(c) $\Theta=\Theta(R)$;

(d) $c+\bar{c}=0$, which restricts the problem to the sourceless case.

We now proceed to examine each case separately.

Case (a): $\Theta=k=$ constant. This is the simplest case, because now Equations (10) and (12) are trivially satisfied. However, the restriction $\Theta=k$ implies $\Lambda=k A+m$ and $M=k P+m$, where $m$ is another constant, which makes $g_{0 \varphi}=(M A-\Lambda P) /$ $W R^{2}=m / R^{2}$. Since $g_{0 \varphi}$ must vanish on the axis we must take $m=0$. Thus $g_{0 \varphi}=0$ everywhere and we have no rotation. Equations (17) and (VI) now determine the equation of state to be $\varrho=$ constant. Finally, the requirement that the equipressure surfaces $R=$ constant be smooth at the axis requires that the constant $g^{2}$ entering in Equation (16) must vanish. Our metric then becomes conformally flat and the conformal factor $R$ a function of the radial coordinate only, so that this case leads to the conformally flat form of Schwarzschild's interior solution.

Case (b): $R=1$. In this case (7) is an identity and (8) and (11) demand that

$$
W=G(z) \bar{G}(\bar{z}), \quad G^{\prime \prime}+g^{2} G=0
$$

where $G$ here is equivalent to $F^{\prime}$ in Equation (16). The general solution of (10) is $\Theta=U(z)+\bar{U}(\bar{z})$ and $(12)$ determines the arbitrary function $U(z)$ in terms of $G(z)$ :

$$
U(z)=\alpha+\beta \frac{G^{\prime}}{G}+\gamma z \frac{G^{\prime}}{G},
$$

where $\alpha$ and $\beta$ are complex and $\gamma$ a real constant. Choosing $G(z)=(\sin g z) / g$ for the solution of (18) which remains finite as $g \rightarrow 0$, and redefining the constants $\alpha$, $\beta, \gamma$ we find the single-variable functions which combine to give $W$ and $\Theta$.

$$
\begin{aligned}
& A=\left(\frac{\sin g \lambda}{g}\right)^{2}+c_{1}, \\
& P=c_{1}-\left(\frac{\sinh g \mu}{g}\right)^{2}, \\
& \Lambda=c_{2}+h\left(\frac{\sin g \lambda}{g}\right)^{2}-m \frac{\sin 2 g \lambda}{g}-r \lambda \sin 2 g \lambda, \\
& M=c_{2}-h\left(\frac{\sinh g \mu}{g}\right)^{2}+q \frac{\sinh 2 g \mu}{g}+r \mu \sinh 2 g \mu,
\end{aligned}
$$

where $h, m, q, r$ are new real constants equivalent to $\alpha+\bar{\alpha}, \beta \pm \bar{\beta}, \gamma$, and $c_{1}$ and $c_{2}$ are separation constants. These constants are restricted by requirements of 
proper behavior on the axis where $M=P=0$. Equations (9) and (VI) now determine the equation of state as $\varrho+3 p=2 g^{2} r=$ constant, suggesting that this is Wahlquist's solution [2]. Indeed, the change of coordinates $\zeta=(\sin g \lambda) / g, \xi=(\sinh g \mu) / g$ shows the equivalence of the two solutions. (See Ref. [2] for more details on this solution.)

Case (c): $\Theta=\Theta(R)$. The assumption $\Theta=\Theta(R)$, together with Equation (16), when substituted in Equations (10) and (12) leads to the system

$$
\begin{aligned}
& R \Theta^{\prime \prime}=2 \Theta^{\prime}, \\
& \Theta^{\prime \prime} F^{\prime 2}+3 \Theta^{\prime} F^{\prime \prime}=\Theta^{\prime \prime} \bar{F}^{\prime 2}+3 \Theta^{\prime} \bar{F}^{\prime \prime}, \\
& F^{\prime \prime}+g^{2} F=c .
\end{aligned}
$$

Remembering that $R=F+\bar{F}$ and $F$ a function of $z$ only, we find that the only solutions of these equations are (i) $\Theta^{\prime}=0$, or (ii) $F^{\prime}= \pm \bar{F}^{\prime}=$ constant. Solution (i) is case $(a)$ while solution (ii) implies $g^{2}=c=0$, which is case $(d)$. Thus this case leads to no new solutions.

Case (d): $c+\bar{c}=0$. (Case without matter.) To obtain solutions different from Carter's [1] we must demand that $R_{z} \neq 0$. A lengthy calculation shows that the most general solution of our equations in this case is

$$
F=i z^{2} / 2+a, \quad R=F+\bar{F}, \quad W=F^{\prime} \bar{F}^{\prime}, \quad \Theta=i b(F-\bar{F})+b\left(a^{2}-1\right),
$$

where $a$ and $b$ are real constants. The functions $A, P, A, M$, and $R$ are given by

$$
\begin{aligned}
& A=\lambda^{2}+1, \quad \Lambda=b\left(\lambda^{2}+1\right)\left(a^{2}-\lambda^{2}\right), \\
& P=1-\mu^{2}, \quad M=b\left(1-\mu^{2}\right)\left(a^{2}+\mu^{2}\right), \\
& R=2(a-\lambda \mu) .
\end{aligned}
$$

Computing the metric, we find that $g_{0 \varphi}=-g_{\varphi \varphi} /\left(a^{2}+1\right)$, so that again we have no rotation $\left(\varphi \rightarrow \varphi^{\prime}+t /\left(a^{2}+1\right)\right.$ makes $\left.g_{0 \varphi}=0\right)$. The coordinate ranges are determined by the requirement that $\Lambda$ and $M$ be positive, $|\mu|<1,|\hat{\lambda}|<|a|$. An examination of the Riemann tensor components reveals that the only singularities of the space occur when $W=\lambda^{2}+\mu^{2}=0$. The zeroes of $\Lambda, M$, and $R$ are coordinate singularities. In fact, all components of the Riemann tensor vanish when $R=0$.

\section{The Case $B=0$}

For completeness, we consider in this section the case $B=0, Q=1$ [see Eq. (1)], in which the canonical separation frame coincides with the comoving frame. As mentioned earlier, we still have the freedom to redefine the coordinate $\lambda$, and we now make use of this freedom to set $\Lambda=1$.

The $G_{1}^{1}-G_{3}^{3}, G_{3}^{2}, G_{1}^{0}$, and $G_{2}^{2}-G_{3}^{3}$ equations determine the $\mu$ dependence of the unknowns as

(i) $R=R(\lambda)$ (independent of $\mu$ ),

(ii) $P=k(1 \pm \mu), k=$ constant,

(iii) $M=l\left(1-\mu^{2}\right), l=$ constant. 
In writing (33) we have changed the origin and scale of $\mu$ to make $M$ vanish at $\mu= \pm 1$ and (32) incorporates the requirement that $P$ vanish ${ }^{4}$ with $M$ (on the axis).

The remaining equations determine $R(\lambda)$ and $A(\lambda)$ when the equation of state is given; they are equivalent to the two propagation equations

$$
\begin{aligned}
2 \frac{R^{\prime \prime}}{R} & =2 \frac{R^{\prime} A^{\prime}}{R A}+\frac{1}{2} \frac{A^{\prime 2}+k^{2}}{A^{2}}+l, \\
\frac{A^{\prime \prime}}{A} & =2 \frac{R^{\prime} A^{\prime}}{R A}+\frac{A^{\prime 2}+k^{2}}{A^{2}}+l-A^{2} f^{\prime}(x),
\end{aligned}
$$

subject to the initial condition

$$
6 \frac{R^{\prime 2}}{R^{2}}-2 \frac{R^{\prime} A^{\prime}}{R A}-\frac{1}{2} \frac{A^{\prime 2}+k^{2}}{A^{2}}-2 l-\frac{A}{R^{2}} f(x)=0 .
$$

In these equations $x=A R^{2}=\left(g_{00}\right)^{-1}$ and $f(x)$ is an arbitrary function, equivalent to the equation of state and determined by it through the equations

$$
\begin{aligned}
f(x) & =16 \pi p \\
x f^{\prime}(x) & =8 \pi(\varrho+p) .
\end{aligned}
$$

These equations make the integrability condition [Eq. (6)] an identity. It can be verified that the initial condition, Equation (36), continues to hold true if it is true initially and $R$ and $A$ satisfy Equations (34) and (35).

The metric is

$$
d s^{2}=\frac{1}{R^{2}}\left\{\frac{(d t-P d \varphi)^{2}}{A}-A\left[d \lambda^{2}+\frac{d \mu^{2}}{M}+M d \varphi^{2}\right]\right\},
$$

so that the comoving metric on the equipressure surfaces $(\lambda=$ constant $)$ is

$$
d s^{2}=\mathrm{const}\left(\frac{d \mu^{2}}{M}+M d \varphi^{2}\right)=\mathrm{const}\left(\frac{d \vartheta^{2}}{l}+l \sin ^{2} \vartheta d \varphi^{2}\right),
$$

where we put $\mu=\cos \vartheta$. The equipressure surfaces will be closed and smooth on the axis $(\mu= \pm 1)$ only when $l=1$, in which case they will be spheres, so that (39) represents an interior solution with the symmetries of NUT space. However, it cannot be matched to exterior $N U T$, without restricting both metrics to their static cases. This result is not unexpected, for, otherwise, we would have, in an asymptotically flat empty space, a rotating perfect fluid mass with spherical rather than oblate shape, contrary to what we know from Newtonian theory.

4 It will be observed that, whichever sign is chosen in Equation (32), $P$ vanishes at only one of the two roots of $M$. We can make $P$ vanish at both roots if we choose $P=k(1-\mu)$ for $0 \leqq \mu \leqq 1$ and $P=k(1+\mu)$ for $-1 \leqq \mu \leqq 0 ; P$ is then continuous but $P$ changes sign at $\mu=0$. However, as the Einstein tensor contains $P^{\prime 2}$ only, the discontinuity in $P^{\prime}$ does not introduce any new distributions of matter. 


\section{Summary}

Starting from a stationary and axisymmetric metric and imposing the requirement that the Hamilton-Jacobi equation for the null geodesics be soluble by separation of variables, we obtained, apart from static or vacuum solutions, two types of solutions representing rotating perfect fluid masses: (a) a general type, admitting an arbitrary equation of state, in which a canonically defined separation frame coincides with the comoving frame; and (b) a particular type, with equation of state $\varrho+3 p=$ constant, with distinct separation and comoving frames. The equipressure surfaces of (a) are spherical and of (b) prolate [2], suggesting that neither can be matched to empty and asymptotically flat exterior solutions. Except for the conformally flat interior Schwarzschild, the solutions obtained are Petrov type $D$.

\section{References}

1. Carter, B.: Commun. math. Phys. 10, 280-310 (1968)

2. Wahlquist, H. D.: Phys. Rev. 172, 1291-1296 (1968)

3. Carter, B.: J. Math. Phys. 10, 70-81 (1969)

4. Zeldovich, Ya. B., Novikov, I. D.: Relativistic Astrophysics, Vol. 1, Section 10.7. Chicago, Ill.: The University of Chicago Press 1971

5. Trümper, M.: Z. f. Naturforschung 22A, 1347-1351 (1968), Eq. 16

6. Chandrasekhar, S., Friedmann, J.L.: Ap. J. 175, 379-405 (1972), Eq. 81

Communicated by J. Ehlers

Received June 28, 1975; in revised form October 6, 1975 
